\title{
Determination of the $b$-quark mass at LEP and SLC
}

\section{Philip Bambade*}

LAL, Université Paris-Sud, Bâtiment 200, B.P. 34, F-91898 Orsay Cédex, France E-mail: 'bambade@lal.in2p3.fri

\section{Maria-Jose Costa, Juan Fuster and Pablo Tortosa}

IFIC, Univ. València-CSIC, Apdo. 22085, E-46071, València, Spain E-mail: 'costa@ific uv.es, fuster@ific uv.es,tortosa@ific.uv.es!

ABSTRACT: Several hadronic event shape observables at LEP and SLC are sensitive to the $b$-quark mass through the reduced gluon radiation which occurs compared to lighter flavours. Detailed measurements by ALEPH, DELPHI, OPAL and SLD collaborations at $\sqrt{s}=M_{Z}$ have established the effect and have enabled determinations of the $b$-quark mass at this energy scale. The most recent results are presented and discussed.

\section{Introduction}

In the Standard Model (SM), spontaneous electroweak symmetry breaking enables fermions to have masses, but the corresponding values are not specified, and remain as free parameters to be measured. Determinations are more difficult for quarks than for charged leptons, because confinement prevents direct observation. Information about masses and other properties must be inferred through phenomenological models, from measurements involving all final state particles. For the $b$-quark mass, the most precise values are obtained at production threshold, extracted from $\Upsilon$ bound states via QCD sum rules and lattice calculations [i] $\left[\begin{array}{l}1 \\ 1\end{array}\right]$. Thanks to the large event samples registered at LEP and SLC by ALEPH, DELPHI, OPAL and SLD experiments, and to the good discrimination available between $b$ and light flavours, it is also possible to probe $b$-quark mass effects at $\sqrt{s}=M_{Z}$,

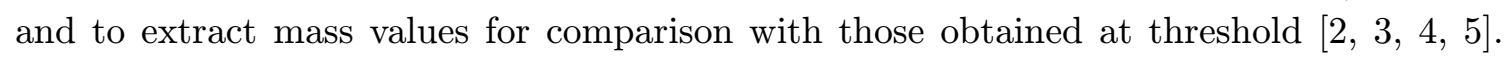
Mass corrections to the $Z \rightarrow b \bar{b}$ coupling are too small for this, being suppressed by $m_{b}^{2} / M_{Z}^{2} \leq 0.003$. But differential quantities like multijet cross-sections can be used, since corresponding corrections are enhanced as $m_{b}^{2} / M_{Z}^{2} / y_{c}$, where $y_{c}<<1$ is the jet resolution parameter. The main physical effect which occurs is a slight reduction in gluon radiation off $b$-quarks compared to lighter flavours, mainly in the collinear region.

\footnotetext{
${ }^{*}$ Speaker.
} 
These measurements are interesting because performed at higher energy and with different methods. Relating values obtained at threshold and at $\sqrt{s}=M_{Z}$ enables to check basic QCD features, such as the predicted evolution. Moreover, in some theories beyond the SM where additional symmetries are postulated, the running of fermion masses can be different. Precise enough measurements of the $b$-quark mass could help to probe these models. In this report, after describing the experimental strategy, the most recent results obtained by the four collaborations are presented. The main systematic limitations are discussed, also highlighting relevant specific aspects emphasized in the different studies.

\section{Experimental strategy}

Quark masses are defined in perturbative QCD in two main ways : the pole mass, $M_{q}$, and the running mass, $m_{q}\left(Q^{2}\right)$, where $Q^{2}$ is the relevant energy scale. They enter into the lagrangian as additional couplings, like the strong coupling constant, $\alpha_{S}$, important to describe observables with sizeable mass effects. Used in the on-shell and $\overline{M S}$ renormalization schemes, respectively, they are equivalent at infinite orders, but their convergence properties vary with the observable.

In recent years, Next-to-Leading Order (NLO) analytical calculations including masses were performed for the ratios of $b$ to light quarks in the case of several event shape observ-

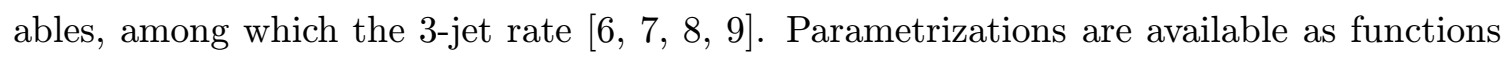
of $\alpha_{S}, \mu$ (the residual renormalization scale dependance in the calculation), $y_{c}$ (in the case of the 3 -jet rate), and the $b$-quark mass, using both pole and running mass definitions.

Experimentally, such ratios were measured by separating events with $b$-quarks from those with light flavours through methods exploiting the longer $B$ hadrons lifetime. Such " $b$-tagging" tools were developed in each experiment thanks to micro-vertex detectors able to resolve the tracks from the displaced $B$ hadron decay vertices. To allow comparison with the analytical results, the measurements had to be expressed in terms of partons. The experimental acceptance (detector coverage, resolution, and flavour selection) was accounted for through corrections computed with the simulation. Non-perturbative effects in the transition from partons to final state hadrons were evaluated with event generators. Comparing the corrected experimental and analytical results enabled both to test the flavour independance of $\alpha_{S}$, by fixing the $b$-quark mass, and determine the $b$-quark mass, by assuming $\alpha_{S}$ universality. The challenge consisted in choosing observables with maximal sensitivity to the $b$-quark mass, while keeping under control systematic uncertainties from the analytical calculations and correction procedures.

\section{ALEPH [2]}

ALEPH studied the normalised ratios of $b$ to light $(u d s)$ flavours for the 3 -jet rate $\left(R_{3}^{b l}\right)$, and for the $1 s t$ and $2 n d$ moments of several shape variables $\left(T, C, y_{3}, B_{T}\right.$ and $\left.B_{W}\right)$. Jets were clustered with the Durham algorithm. Only the 3 -jet rate and the 1 st moment of the 3 -to-2 jets transition value, $y_{3_{1}}$, had both small hadronization corrections and NLO contributions (using pole and running mass definitions), and were therefore prefered to 
minimize corresponding uncertainties. The best results were obtained using $y_{3_{1}}$ :

$$
\begin{aligned}
m_{b}\left(M_{Z}\right) & =3.27 \pm 0.22(\text { stat }) \pm 0.22(\exp ) \pm 0.38(\text { had }) \pm 0.16(\text { theo }) \mathrm{GeV} / \mathrm{c}^{2} \\
M_{b} & =4.73 \pm 0.29(\text { stat }) \pm 0.29(\exp ) \pm 0.49(\text { had }) \pm 0.18(\text { theo }) \mathrm{GeV} / \mathrm{c}^{2} \\
\alpha_{S}^{b} / \alpha_{S}^{l} & =0.997 \pm 0.004(\text { stat }) \pm 0.004(\exp ) \pm 0.007(\text { had }) \pm 0.003(\text { theo })
\end{aligned}
$$

The largest systematic error (labelled "had") came from modeling the hadronization. It was evaluated by propagating uncertainties in the experimental tuning of generator parameters through the correction procedure, by studying effects from changing the parametrization of the $b$-quark fragmentation function, and by comparing corrections in the main generator used, PYTHIA/JETSET (where hadronization is treated by the string model) to those in an alternative program, HERWIG (based on the cluster model). To reduce potential biases in the correction procedure, the probability of gluon splittings into $b \bar{b}$ was close to doubled in the simulation, to match measured rates. The experimental uncertainty ("exp") reflects mostly observed discrepancies in the simulation of the vertex detector used in the b-tagging algorithm. The theoretical uncertainty ("theo") represents estimates of higher orders missing beyond NLO in the analytical expressions used to extract the $b$-quark mass, computed by changing the renormalisation scale and mass definitions used.

\section{DELPHI [3]}

DELPHI focussed its study on $R_{3}^{b l}$, using two jet clustering algorithms, Durham and Cambridge, and computations with both pole and running mass definitions. Corresponding

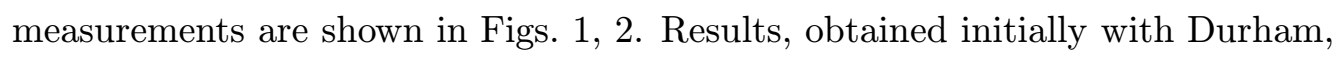

$$
\begin{aligned}
m_{b}\left(M_{Z}\right) & =2.67 \pm 0.25(\text { stat }) \pm 0.34(\text { had }) \pm 0.27(\text { theo }) \mathrm{GeV} / \mathrm{c}^{2} \\
\alpha_{S}^{b} / \alpha_{S}^{l} & =1.007 \pm 0.005(\text { stat }) \pm 0.007(\text { had }) \pm 0.005(\text { theo }),
\end{aligned}
$$

and, more recently, with Cambridge,

$$
\begin{aligned}
m_{b}\left(M_{Z}\right) & =2.61 \pm 0.18(\text { stat }) \pm 0.47(\text { had }) \pm 0.18(\operatorname{tag}) \pm 0.12(\text { theo }) \mathrm{GeV} / \mathrm{c}^{2} \\
M_{b} & =4.1 \pm 0.5(\text { stat }+ \text { had }+ \text { theo }) \mathrm{GeV} / \mathrm{c}^{2} \\
\alpha_{S}^{b} / \alpha_{S}^{l} & =1.005 \pm 0.012(\text { stat }+ \text { had }+ \text { theo })
\end{aligned}
$$

are compatible. Theory and hadronization errors were evaluated similarly to ALEPH. The running mass definition was advantageous for Cambridge, because the NLO correction was smaller (see Fig. '2i $2 \bar{i}$ ), and the theoretical uncertainty reduced [9i]. A new error (labelled "tag") was evaluated in the $2 n d$ measurement, comparing two $b$-tagging methods used in DELPHI. But, as in ALEPH, the dominating uncertainties were from the hadronization. Recently, its main component, derived comparing corrections in PYTHIA/JETSET and HERWIG, was studied as a function of the subleading $b$-jet scaled energy, an observable partly correlated to the $B$-hadron energy. Corrections in both generators were much closer 
when the smallest values for this quantity were avoided. This pointed to differences mainly in tails of the corresponding distributions, where accurate description with present generators and tuning methods may be harder. Promising improvements are expected by restricting the measurement to this region, and from studies of these tails.

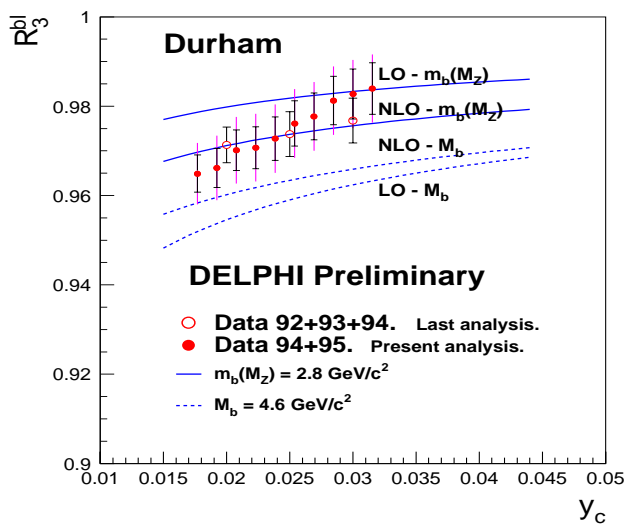

Figure 1: $R_{3}^{b l}$ with Durham.

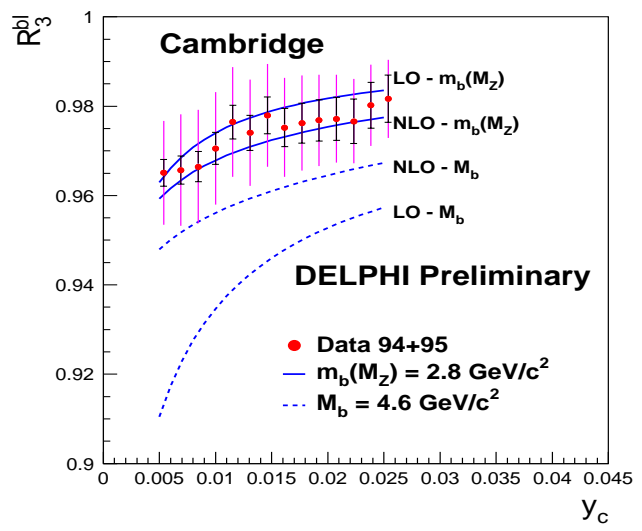

Figure 2: $R_{3}^{b l}$ with Cambridge.

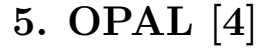

The first OPAL study measured several shape variables $\left(T, C, y_{3}, M_{H}\right.$ and $\left.B_{W}\right)$ for $b, c$ and light flavours and extracted simultaneusouly $\alpha_{S}^{b} / \alpha_{S}^{u d s}, \alpha_{S}^{c} / \alpha_{S}^{u d s}$ and $\alpha_{S}^{u d s}$ from a 3 parameter fit to massive NLO calculations, resulting in

$$
\begin{aligned}
& \alpha_{S}^{b} / \alpha_{S}^{l}=0.993 \pm 0.008(\text { stat }) \pm 0.006(\text { syst }) \pm 0.011(\text { theo }) \\
& \alpha_{S}^{c} / \alpha_{S}^{l}=0.997 \pm 0.038(\text { stat }) \pm 0.030(\text { had }) \pm 0.012(\text { theo })
\end{aligned}
$$

Recently, seven jet clustering algorithms (Durham, Geneva, Jade, and its variants E, E0, $\mathrm{P}, \mathrm{P} 0$ ) were also used to measure $R_{3}^{b l}$ (where here $l$ includes also the $c$ flavour). Values extracted for $m_{b}\left(M_{Z}\right)$ are shown in Fig. ${ }^{-3}$. Jade E and E0 algorithms had the best statistical sensitivity, but also significant systematics. The main effects were from missing higher orders in the calculations and from hadronization corrections. The latter were evaluated exclusively with PYTHIA/JETSET, propagating uncertainties from the parameter tuning, and trying different parametrizations for the $b$-quark fragmentation function. They dominated most of the measurements, although large effects from the detector simulation also affected some of them. A combined result with a very small statistical error was obtained minimising their $\chi^{2}$ with correlations included, and propagating systematic errors :

$$
m_{b}\left(M_{Z}\right)=2.67 \pm 0.03(\text { stat })_{-0.37}^{+0.29}(\text { syst }) \pm 0.19(\text { theo }) \mathrm{GeV} / \mathrm{c}^{2} .
$$


A technical limitation from the generator was addressed. After hadronization, there was no dependance on the input mass, because the scheme used to generate fixed final $B$-hadron masses absorbed resulting changes in the radiation. Although artificial, this feature of the simulation could imply arbitrariness in all results, since measured values would depend on the choice of input $b$-quark mass, via the corrections. To avoid this contradiction, OPAL used a version of the simulation with $b$-quark masses changed only in the 1 st gluon emmission, and showed that effects then carried over to the hadron level.

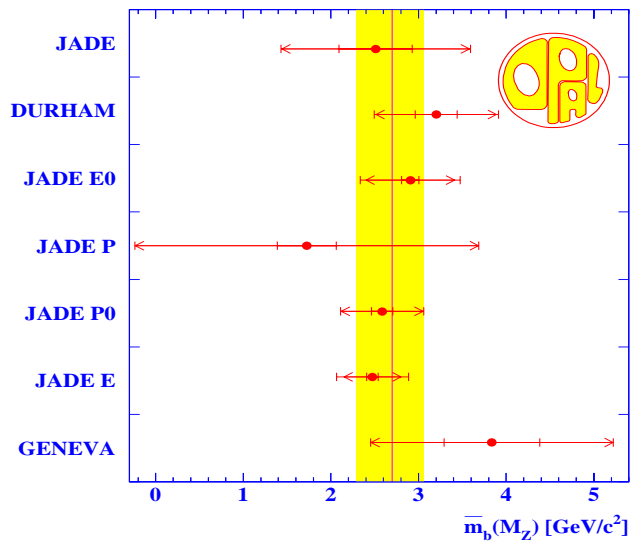

Figure 3: $R_{3}^{b l}$ with 7 jet algorithm.

\section{SLD [5]}

SLD and Brandenburg et al. used six jet clustering algorithms (Durham, Geneva, and the four Jade variants E, E0, P, P0) to measure $R_{3}^{b l}$ and $R_{3}^{c l}$. NLO calculations were used to test the flavour independance of $\alpha_{S}$, fixing $m_{b}\left(M_{Z}\right)=3 \mathrm{GeV} / \mathrm{c}^{2}$. Results were compatible, and combined into single ones,

$$
\begin{aligned}
& \alpha_{S}^{b} / \alpha_{S}^{l}=1.004 \pm 0.018(\text { stat })_{-0.031}^{+0.026}(\text { syst })_{-0.029}^{+0.018}(\text { theo }), \\
& \alpha_{S}^{c} / \alpha_{S}^{l}=1.036 \pm 0.043(\text { stat })_{-0.045}^{+0.041}(\text { had })_{-0.018}^{+0.020}(\text { theo }),
\end{aligned}
$$

through weighted averages, conservatively assuming 100\% correlation. Systematic effects, mainly from missing higher orders in the calculations and from hadronization corrections, were evaluated in ways similar to ALEPH and DELPHI. The same measurements were also interpreted in terms of $m_{b}\left(M_{Z}\right)$. The spread of values obtained was however larger than expected for such strongly correlated measurements, suggesting some unaccounted source of uncertainty. A $\chi^{2}$ minimisation similar to OPAL's gave acceptable results only upon introducing by hand additional uncorrelated errors of about $0.5 \mathrm{GeV} / \mathrm{c}^{2}$. Under the assumption that hadronization uncertainties are independent of the jet algorithm, the main source of additional error was suspected to be from missing higher orders in the calculations, not fully represented by the evaluation done. This could be the case, particularly for the set of Jade algorithms used : although more sensitive to the $b$-quark mass, they are known to have significantly worse soft gluon behaviour than Durham or Geneva, which could result in slower convergence for the corresponding perturbative expansions. However, in the absence of a conclusive proof, no algorithm was excluded. The final result,

$$
m_{b}\left(M_{Z}\right)=2.56 \pm 0.27 \text { (stat) }{ }_{-0.38}^{+0.28}(\text { syst })_{-1.48}^{+0.49}(\text { theo }) \mathrm{GeV} / \mathrm{c}^{2},
$$

conservatively incorporated the added uncorrelated $0.5 \mathrm{GeV} / \mathrm{c}^{2}$ error in the combination. 


\section{Summary and conclusion}

Effects from the $b$-quark mass on event shapes at LEP and SLC were measured. Massive NLO calculations enabled tests of the flavour independance of $\alpha_{S}$ at the $1 \%$ level, and determinations of $m_{b}\left(M_{Z}\right)$ within a few hundred $\mathrm{MeV}$. Results were compatible (see Fig. 'ili) and agreed with threshold values extrapolated to $M_{Z}$ by the predicted QCD evolution. Observables used had different sensitivity and systematics. All four studies illustrated the advantage of observables computed with small high order corrections. However, hadroniza-

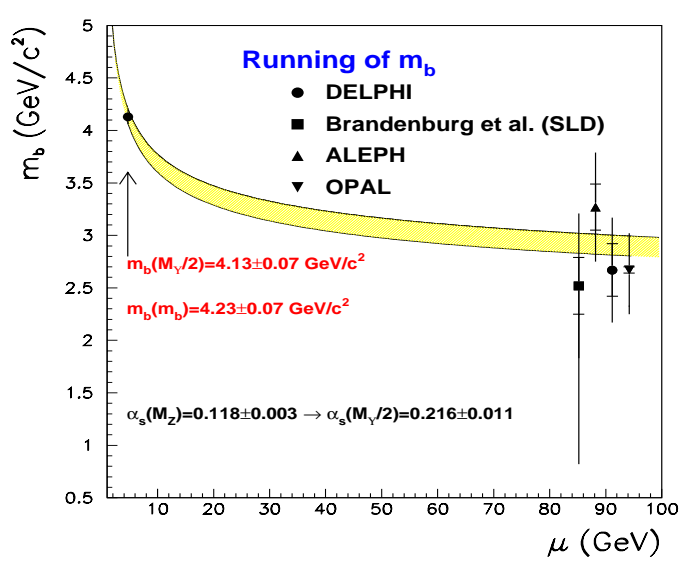

Figure 4: Measured values of $m_{b}(\mu)$. tion uncertainties tended to dominate, and were generally evaluated in similar ways. Some important aspects were also uncovered separately. ALEPH took into account biases from the rate of gluon splittings into $b \bar{b}$. DELPHI identified a kinematical region with small hadronization uncertainty. OPAL showed how the contradictory behaviour of the $b$-quark mass as a parameter in the generator could be avoided, using a modified version where effects from varying the mass are not artificially absorbed in the hadronization. Thanks to the recent progress, results are improving. A common consideration of the different effects could help, and might enable meaningful combination of the results. Futher reductions in hadronization uncertainties may also be possible by improving the tuning of generator parameters, including $b$-quark mass effects, and by taking into account correlations in their errors

\section{References}

[1] M. Jamin and A. Pich Nucl. Phys. B507 (1997), 334.

V. Giménez et al. JHEP 0003:018 (2000)

[2] ALEPH Collaboration, R. Barate et al., Eur. Phys. J. C18 (2000) 1.

[3] DELPHI Collaboration, P. Abreu et al., Phys. Lett. B418 (1998) 430.

DELPHI Collaboration, An improved determination of the b-quark mass by DELPHI, Contribution to the HEP conference in Osaka, Japan, 2000, DELPHI 2000-121 CONF 420.

[4] OPAL Collaboration, G. Abbiendi et al., Eur. Phys. J. C11 (1999) 643. OPAL Collaboration, G. Abbiendi et al., Eur. Phys. J. C21 (2001) 411.

[5] SLD Collaboration, K. Abe et al., Phys. Rev. D59 (1999) 12002.

A. Brandenburg et al., Phys. Lett. B468 (1999) 168.

[6] G. Rodrigo et al., Phys. Rev. Lett. 79 (1997) 193.

[7] W. Bernreuther et al., Phys. Rev. Lett. 79 (1997) 189.

[8] P. Nason et al., Phys. Rev. Lett. B407 (1997) 57.

[9] M. Bilenkii et al., Phys. Rev. D60 (1999) 114006. 